Wójcik Magdalena, Dąbrowska Justyna, Boreński Grzegorz, Bator Damian, Milanowska Joanna. Gut microbiota and its impact on neuropsychiatric disorders. Journal of Education, Health and Sport. 2020;10(8):276-285. eISSN 2391-8306. DOI http://dx.doi.org/10.12775/JEHS.2020.10.08.032

https://apcz.umk.pl/czasopisma/index.php/JEHS/article/view/JEHS.2020.10.08.032

https://zenodo.org/record/3992560

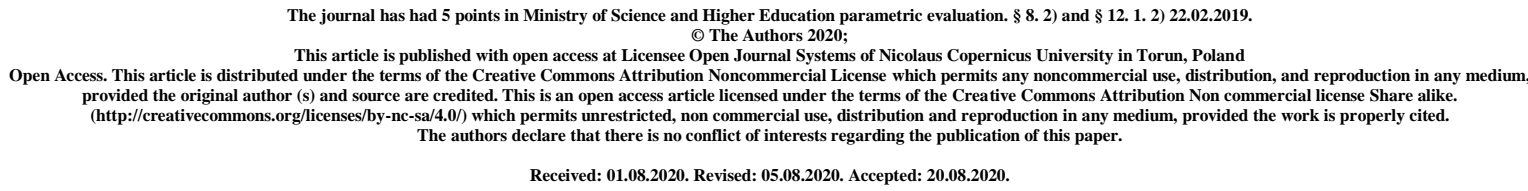

\title{
Gut microbiota and its impact on neuropsychiatric disorders
}

\author{
Magdalena Wójcik $^{1 *}$, Justyna Dąbrowska ${ }^{1}$, Grzegorz Boreński ${ }^{1}$, Damian Bator ${ }^{1}$, \\ Joanna Milanowska ${ }^{2}$ \\ ${ }^{1}$ Student Science Club at the Department of Applied Psychology, Medical University of \\ Lublin, Lublin, Poland \\ ${ }^{2}$ Department of Applied Psychology, Medical University of Lublin, Lublin, Poland \\ *E-mail address: magdalena.wojcik967@gmail.com
}

\section{ORCID ID:}

Magdalena Wójcik https://orcid.org/0000-0002-0999-6284

Justyna Dąbrowska https://orcid.org/0000-0002-1356-6965

Damian Bator https://orcid.org/0000-0002-8464-932X

Joanna Milanowska https://orcid.org/0000-0001-9741-1583

\begin{abstract}
Introduction: Over the last few decades, the interest of microbiota- gut - brain axis has increased. It is thought to have an impact on the overall health of the host. Gut microbiota and central nervous system can communicate in different ways. The connection between them is especially interesting in case of neuropsychiatric diseases

The aim of the study: The purpose of this systemic review was to collect and analyse current data of the association between gut microbiota and neuropsychiatric disorders, specifically schizophrenia and bipolar disorder
\end{abstract}


Material and method: Standard criteria were used to review the literature data. The search of articles in the PubMed and Google Scholar database was carried out using the following keywords: gut microbiota, dysbiosis, schizophrenia, bipolar disorder

Description of the state of knowledge: Dysregulation of microbiota is a potential factor in pathogenesis of brain disorders. Gut microbiome is thought to be an important part of not only normal brain development, but also modulation of host physiological systems. Studies show that the composition of microbiota in psychiatric diseases including schizophrenia and bipolar disorder differs from healthy individuals. Many environmental factors can change the composition of microbiota and therefore potentially contribute to the development of many disorders, including neuropsychiatric disorders.

Summary: Microbiota is a potential factor contributing to the onset or modulating the course of many illnesses, including brain disorders The subject of imbalanced microbiota composition give hope for finding etiology or improving treatment of many disorders. However, it still requires more studies.

\section{Key words: gut microbiota, dysbiosis, schizophrenia, bipolar disorder}

\section{Introduction}

The number of bacteria in an adult's body is estimated at 100 trillion, $80 \%$ of which can be found in the gut [1]. Human body contains ten times more microbial cells than human cells. That is because of extremely high density of microbes found in the human intestinal tract (typically $10^{11}-10^{12}$ microbes $/ \mathrm{ml}$ of luminal content) [2]. Alterations in bidirectional brain-gut microbiota interactions are believed to be involved in the pathogenesis of irritable bowel syndrome [3,4] and gastrointestinal disorders [5]. Dysregulation of microbiota is a potential factor in pathogenesis of brain disorders such as mood disorders [6,7] and autism spectrum disorder (ASD) [8]. Altered microbiota was also associated with eating disorders $[9,10]$ and Parkinson's disease (PD) [11]. Intestinal microbiota is also a potential factor in balancing the HPA axis. intestinal microbes can be a reason of imbalances of the HPA axis affecting the neuroendocrine system in the brain, which can lead to anxiety-like behavioral phenotype [12]. The gut microbiome can induce beneficial effects on host health and lifespan depending on the host nutrient signaling pathways. Although gut dysbiosis disturb the interdependence leading to reduction of the beneficial effects or even reverse effects. It can also trigger the innate immune response and chronic low-grade inflammation [13]. It is now known that a healthy gut microbiota has a large impact on the overall health of the host [14]. There is a number of common features between schizophrenia and bipolar disorder, both symptomatically and biologically [15].Alterations of the gut microbiom composition were also fund within patients with bipolar disorder [16] and schizophrenia [17].

\section{The Gut - Brain Axis}

A huge amount of bacteria, archaea, viruses, and unicellular eukaryotes inhabit human body. Those coexisting microorganisms are known as microbiota [18]. The presence of the microbiota differs within different parts of the gastrointestinal tract. In the stomach and small intestine few micro-organisms can be found. 
However, in the colon there is a concentration of approximately 1.012 bacteria, with two main representations of Firmicutes and Bacteriodetes phyla [19]. Microbiota is known to have an important role in immune system [20, 21], the host metabolism [22, 23] and is increasingly recognized for influencing the development of host nervous system and behaviors [24].

Central nervous system (CNS) sends regulatory signals to the gut and vice versa [25]. The connection between the gut and the brain exists on many pathways including:

- $\quad$ the enteric nervous system (ENS)

- $\quad$ vagus nerve

- $\quad$ the immune system

- $\quad$ the metabolic processes of gut microorganisms [26].

The motor, sensory, and secretory modalities of gastrointestinal tract may be influenced by the signals coming from the brain, but also visceral signals from gastrointestinal tract are able to influence brain function [27]. The autonomic system leads afferent signals emerging from the lumen, transmitted through enteric, spinal and vagal pathways to CNS, but also the efferent signals form CNS to the intestinal wall [28]. The vagus nerve (VN) is the tenth cranial nerve. $\mathrm{VN}$ contains approximately $80 \%$ afferent and $20 \%$ efferent fibers [29]. It is thought to be an important component in the communication pathway between the bacteria exposed to the gut and the brain [30]. The afferent fibers of vagus nerve are crucial for conveying visceral information to the brain [31]. Immune-related and neural signals coming from bacteria, including 'probiotic' organisms, are transmitted from the gut through blood circulation or directly via the vagus nerve to the CNS [32]. Vagus nerve is thought to be able to dampen peripheral inflammation and to decrease intestinal permeability due to the cholinergic anti-inflammatory pathway that was represented through its fibers [33].The gut microbiota is linked with the immune system due to its ability of the commensal microbiome to regulate the maturation of the mucosal immune system. However, the pathogenic microbiome causes immunity dysfunction [34]. Another function of microbiota in immune system is its ability to influence the activation of peripheral immune cells that regulate responses to neuroinflammation, brain injury, autoimmunity and neurogenesis [35]. It has been suggested that composition of gastrointestinal microbiota has a role in resilience to stress- and immune-related disorders and dysfunction of stress- and immune-systems [36]. Gram-negative bacteria can bind of the lipopolysaccharide (LPS) component of their cell walls to toll-like receptors present at monocytes, macrophages and microglia and through this stimulate production of pro-inflammatory cytokines [37]. The immune system of host is thought to be able to sense gut bacterial metabolites in addition to pathogen-associated molecular patterns (PAMP). Moreover, recognition of those can influence the host immune response concerning disease and inflammation in the gut and beyond [38]. Short-chain fatty acids (SCFA) are the major end products of bacterial degradation of soluble fiber in the large intestine. The main short-chain fatty acids produced during this process are acetale, propionate and butyrate. SCFA are a group of bacterial metabolites with pleiotropic effects on host immune and energy state [39]. It is well known that SCFA, especially butyrate, play an import ant role in maintaining the colonic epithelium [40]. SCFA may reduce migration and proliferation of immune cells, decrease many types of cytokines and cause apoptosis, thus suppress inflammation. 
Nevertheless, clear alterations of SCFA concentrations in blood or various tissues may be the reason for disorders related to immunological and metabolic imbalances [41].

\section{Gut microbiota and neuropsychiatry}

Gut microbiome is thought to be an important part of not only normal brain development, but also modulation of host physiological systems which are significant in stress-related disorders [42]. Emotional and physiological stress influence the composition of gut microbiota [43]. Moreover, it has been suggested that gut microbiota affects brain function via the inflammasome signalling platform which influence inflammatory pathways altering brain function and due to this affecting depressive- and anxiety-like behaviours [44].

"Developmental programming" is a process in which environmental factor occurs in a sensitive or vulnerable developmental period, especially In early life, influencing structure and function of organs which may last throughout life. One of these factors is gut microbiota [45]. Factors like caesarean section, formula feeding and early-life antibiotic exposures can influence microbiome establishing and potentially affect health of the host [46].

In case of many neuropsychiatric diseases it is still not fully understood what causes them. Without knowing the accurate biological target of those diseases, it makes it difficult to treat them successfully [47].

Gut microbiota has influence on many aspects of host's functioning. The study by Manderino et.al (2017) performed on neurologically-healthy older adults suggests association between composition of the gut microbiome cognitive test performance. In this study participated 43 older adults. Tests of their stool samples showed different distributions of Bacteroidetes, Firmicutes, Proteobacteria and Verrucomicrobia compering Intact and Impaired groups [48]. Another aspect of gut microbiota is the association with mood disorders. Stressors and pathogens can cause inflammatory responses either exaggerated or prolonged which is an important factor in depression's pathogenesis for a subset of depressed individuals [49]. Other factor that increase inflammatory responses is intestinal permeability caused by depression, childhood adversity, stressors, and diet [49]. Bacteria in the genus Lactobacillus which increase the health of the host, during stress is consistently reduced [50]. It has been suggested that gut microbiota plays a role in balancing the hypothalamic-pituitary-adrenal (HPA) axis and if imbalance occurs it can lead to an anxiety-like behavioral phenotype [51]. Studies in animals showed that gut bacteria influence behavior, Brain-Derived Neurotrophic Factor (BDNF) levels and serotonin metabolism [52]. In the study by Sharon (2019) gut microbiota from human donors with autism spectrum disorder (ASD) or typically-developing (TD) controls was transplanted into germ-free mice. The study showed that ASD and TD microbiota produce differential metabolome profiles in mice. colonization with ASD microbiota is enough to induce characteristic autistic behaviors. Moreover, the administration of specific metabolites is able to correct those behaviors in mice [53]. Gut microbiota is also linked to dietary habits. Changes in dietary fibers can change composition of gutmicrobiota. Diet is a main factor determining composition of the colonic microbiome. However, the host genetic background and the colonic milieu can also influence this composition [54]. It is possible that gut microbiota influence the gut-brain axis in eating disorder altering appetite control and brain function as part of their the genesis [55]. 
Researches show growing evidences that the intestinal microbiota plays a substantial role in nutrient extraction and host metabolism [56].

\section{Schizophrenia and bipolar disorder}

Schizophrenia (SCZ) is a severe psychotic disorder which core features are cognitive impairment, negative and positive symptoms. It affects approximately $1 \%$ of the adult population worldwide over the average lifetime [57,58]. Genetic factors combined with environmental insults such as prenatal infection, perinatal complication, and cannabis use are considered to be the cause of schizophrenia [59]. Bipolar disorder (BD) is a chronic disorder of a recurring character with symptoms of mood state and energy fluctuations. Functional decline, cognitive impairment, and a reduction in quality of life are observed in patients suffering from bipolar disorder [60]. Mood fluctuates between episodes of mood elevation (mania) and depression with interlaced periods of euthymia [61]. BP affects around $2.4 \%$ of the global population [60]. Bipolar disorder and schizophrenia have many features in common such as some of the characteristic symptoms and the lifelong course. Etiology of both diseases is not fully understood and they are classified by their phenotypic features [62].Common genetic etiologies have been found between schizophrenia and bipolar disorder [63]. Severe mental illnesses (SMI), mainly schizophrenia and bipolar disorder (BD), are a leading global cause of disability and they are considered one of the most notable causes of death worldwide [64]. In the study by Zheng et al. (2019) the gut microbial communities of patients with SCZ and healthy controls (HCs) was compared. The study revealed that the microbial composition of patients with SCZ was less diverse than that of HC individuals. There was also an association between microbiota composition of SCZ patients and lower $\alpha$-diversity stores comparing to $\mathrm{HC}$ group. It was explained that a high $\alpha$-diversity is thought to represent a marker of "good" health status. Moreover, results of the study showed unique bacterial taxa linked to SCZ severity. In the same study microbiomes of SCZ group and HC group were transferred to mice. Compared to mice with HC group microbiota, those with human SCZ microbiomes presented changes in gut microbial composition and behavioral phenotypes proper to SCZ. However, those behavioral phenotypes may be nonspecific [65]. The study by Liang et al. (2019) compared the metabolic signatures of the cortex, cerebellum and striatum in schizophrenia microbiota and healthy microbiota recipient mice. Results showed relevant differences of the metabolite signatures between those two groups. Moreover, according to the study disruptions of glycerophospholipid and fatty acyl metabolism were linked to the onset of schizophrenia-related behaviors [66]. In the study by $\mathrm{Hu}$ et al. (2019) the gut microbiota in depressed patients with bipolar disorder before and after quetiapine treatment was characterized and compared with healthy controls. Result showed major differences between $\mathrm{BD}$ and $\mathrm{HC}$ groups. Bacteroidetes and Firmicutes dominated respectively in the gut microbiota composition in untreated BD patients and HCs.Moreover, lower levels of butyrateproducing bacteria was observed in untreated patients. Comparing BD and $\mathrm{HC}$ group, greater diversity of gut micro biota was observed in HCs. Quetiapine treatment caused significant changes in gut microbial composition. Large genera of Klebsiella and Veillonella were observed in treated patients [67]. In the study by Aizawa et al. (2019) fecal samples from patients with bipolar disorder and healthy controls were examined and bacterial counts were compared. 
As the results, there were no significant differences in bacterial counts between those two groups. Although, Lactobacillus counts and sleep showed negative correlation. A negative correlation between Bifidobacterium counts and cortisol levels in patients were found. The results suggests that these bacteria may play a role in sleep and stress response in the patients [68].

\section{Conclusion}

Over the last few decades, gut microbiota has become a subject of interest in many fields of science. The microbiota - gut - brain axis is thought to be involved in immune system, the host metabolism, central nervous system and behaviors. Microbiota is a potential factor contributing to the onset or modulating the course of many illnesses, including brain disorders. Different ways of connection between gut microbiota and central nervous system were described. The connection between them is especially interesting in case of neuropsychiatric diseases. There are many studies on both animals and humans, pointing the potential role of microbiota dysbiosis in psychiatric illnesses. Revealing the etiology of schizophrenia or bipolar disorder would make the treatment of them much more effective. However, the subject of microbiota is still in its infancy and require more studies.

\section{References}

1. Wang HX, Wang YP. Gut Microbiota-brain Axis. Chin Med J (Engl). 2016;129(19):23732380. doi:10.4103/0366-6999.190667

2. Palmer C, Bik EM, DiGiulio DB, Relman DA, Brown PO. Development of the human infant intestinal microbiota. PLoS Biol. 2007;5(7):e177. doi:10.1371/journal.pbio.0050177

3. Rodiño-Janeiro BK, Vicario M, Alonso-Cotoner C, Pascua-García R, Santos J. A Review of Microbiota and Irritable Bowel Syndrome: Future in Therapies. Adv Ther. 2018;35(3):289-310. doi:10.1007/s12325-018-0673-5

4. Raskov H, Burcharth J, Pommergaard HC, Rosenberg J. Irritable bowel syndrome, the microbiota and the gut-brain axis. Gut Microbes. 2016;7(5):365-383. doi:10.1080/19490976.2016.1218585

5. Zhang M, Yang XJ. Effects of a high fat diet on intestinal microbiota and gastrointestinal diseases. World J Gastroenterol. 2016;22(40):8905-8909. doi:10.3748/wjg.v22.i40.8905

6. Jiang $\mathrm{H}$, Ling Z, Zhang $\mathrm{Y}$, Mao $\mathrm{H}, \mathrm{Ma} \mathrm{Z}$ et al. Altered fecal microbiota composition in patients with major depressive disorder. Brain Behav Immun. 2015;48:186-194. doi:10.1016/j.bbi.2015.03.016

7. Huang TT, Lai JB, Du YL, Xu Y, Ruan LM, Hu SH. Current Understanding of Gut Microbiota in Mood Disorders: An Update of Human Studies. Front Genet. 2019;10:98. Published 2019 Feb 19. doi:10.3389/fgene.2019.00098

8. Li Q, Han Y, Dy ABC, Hagerman RJ. The Gut Microbiota and Autism Spectrum Disorders. Front Cell Neurosci. 2017;11:120. Published 2017 Apr 28. doi:10.3389/fncel.2017.00120

9. Borgo F, Riva A, Benetti A, Casiraghi MC, Bertelli S et al. Microbiota in anorexia nervosa: The triangle between bacterial species, metabolites and psychological tests. PLoS One. 2017;12(6):e0179739. Published 2017 Jun 21. doi:10.1371/journal.pone.0179739

10. Kleiman SC, Watson HJ, Bulik-Sullivan EC, Huh EY, Tarantino LM et al. The Intestinal Microbiota in Acute Anorexia Nervosa and During Renourishment: Relationship to Depression, 
Anxiety, and Eating Disorder Psychopathology. Psychosom Med. 2015;77(9):969-981. doi:10.1097/PSY.0000000000000247

11. Mulak A, Bonaz B. Brain-gut-microbiota axis in Parkinson's disease. World J Gastroenterol. 2015;21(37):10609-10620. doi:10.3748/wjg.v21.i37.10609

12. Huo R, Zeng B, Zeng L, Cheng K, Li B et al. Microbiota Modulate Anxiety-Like Behavior and Endocrine Abnormalities in Hypothalamic-Pituitary-Adrenal Axis. Front Cell Infect Microbiol. 2017;7:489. Published 2017 Nov 30. doi:10.3389/fcimb.2017.00489

13. Kim S, Jazwinski SM. The Gut Microbiota and Healthy Aging: A Mini-Review. Gerontology. 2018;64(6):513-520. doi:10.1159/000490615

14. Jandhyala SM, Talukdar R, Subramanyam C, Vuyyuru H, Sasikala M, Nageshwar Reddy D. Role of the normal gut microbiota. World $J$ Gastroenterol. 2015;21(29):8787-8803. doi:10.3748/wjg.v21.i29.8787

15. Fillman SG, Sinclair D, Fung SJ, Webster MJ, Shannon Weickert C. Markers of inflammation and stress distinguish subsets of individuals with schizophrenia and bipolar disorder. Transl Psychiatry. 2014;4(2):e365. Published 2014 Feb 25. doi:10.1038/tp.2014.8

16. Evans SJ, Bassis CM, Hein R, Assari S, Flowers SA et al. The gut microbiome composition associates with bipolar disorder and illness severity. $J$ Psychiatr Res. 2017;87:23-29. doi:10.1016/j.jpsychires.2016.12.007

17. Castro-Nallar E, Bendall ML, Pérez-Losada M, Sabuncyan S, Severance EG et al. Composition, taxonomy and functional diversity of the oropharynx microbiome in individuals with schizophrenia and controls. PeerJ. 2015;3:e1140. Published 2015 Aug 25. doi:10.7717/peerj.1140

18. Tang WH, Kitai T, Hazen SL. Gut Microbiota in Cardiovascular Health and Disease. Circ Res. 2017;120(7):1183-1196. doi:10.1161/CIRCRESAHA.117.309715

19. Mangiola F, Ianiro G, Franceschi F, Fagiuoli S, Gasbarrini G, Gasbarrini A. Gut microbiota in autism and mood disorders. World J Gastroenterol. 2016;22(1):361-368. doi:10.3748/wjg.v22.i1.361

20. Belkaid Y, Harrison OJ. Homeostatic Immunity and the Microbiota. Immunity. 2017;46(4):562-576. doi:10.1016/j.immuni.2017.04.008

21. Gao J, Xu K, Liu H, Liu G, Bai M et al. Impact of the Gut Microbiota on Intestinal Immunity Mediated by Tryptophan Metabolism. Front Cell Infect Microbiol. 2018;8:13. Published 2018 Feb 6. doi:10.3389/fcimb.2018.00013

22. Staley C, Weingarden AR, Khoruts A, Sadowsky MJ. Interaction of gut microbiota with bile acid metabolism and its influence on disease states. Appl Microbiol Biotechnol. 2017;101(1):47-64. doi:10.1007/s00253-016-8006-6

23. Boulangé CL, Neves AL, Chilloux J, Nicholson JK, Dumas ME. Impact of the gut microbiota on inflammation, obesity, and metabolic disease. Genome Med. 2016;8(1):42. Published 2016 Apr 20. doi:10.1186/s13073-016-0303-2

24. Vuong HE, Yano JM, Fung TC, Hsiao EY. The Microbiome and Host Behavior. Annu Rev Neurosci. 2017;40:21-49. doi:10.1146/annurev-neuro-072116-031347

25. Wang Y, Kasper LH. The role of microbiome in central nervous system disorders. Brain Behav Immun. 2014;38:1-12. doi:10.1016/j.bbi.2013.12.015

26. Zhu X, Han Y, Du J, Liu R, Jin K, Yi W. Microbiota-gut-brain axis and the central nervous $\begin{array}{llllll}\text { system. Oncotarget. 2017;8(32):53829-53838. Published } 2017 & \text { May } & 10 .\end{array}$ doi:10.18632/oncotarget.17754

27. Grenham S, Clarke G, Cryan JF, Dinan TG. Brain-gut-microbe communication in health and disease. Front Physiol. 2011;2:94. Published 2011 Dec 7. doi:10.3389/fphys.2011.00094

28. Carabotti M, Scirocco A, Maselli MA, Severi C. The gut-brain axis: interactions between enteric microbiota, central and enteric nervous systems. Ann Gastroenterol. 2015;28(2):203-209. 
29. Bonaz B, Sinniger V, Pellissier S. Anti-inflammatory properties of the vagus nerve: potential therapeutic implications of vagus nerve stimulation. $J$ Physiol. 2016;594(20):5781-5790. doi:10.1113/JP271539

30. Bravo JA, Forsythe P, Chew MV, Escaravage E, Savignac HM et al. Ingestion of Lactobacillus strain regulates emotional behavior and central GABA receptor expression in a mouse via the vagus nerve. Proc Natl Acad Sci $U$ S A. 2011;108(38):16050-16055. doi:10.1073/pnas.1102999108

31. Klarer M, Arnold M, Günther L, Winter C, Langhans W, Meyer U. Gut vagal afferents differentially modulate innate anxiety and learned fear. J Neurosci. 2014;34(21):7067-7076. doi:10.1523/JNEUROSCI.0252-14.2014

32. Poutahidis T, Kearney SM, Levkovich T, Qi P, Varian BJ et al. Microbial symbionts accelerate wound healing via the neuropeptide hormone oxytocin. PLoS One. 2013;8(10):e78898. Published 2013 Oct 30. doi:10.1371/journal.pone.0078898

33. Bonaz B, Bazin T, Pellissier S. The Vagus Nerve at the Interface of the Microbiota-Gut-Brain Axis. Front Neurosci. 2018;12:49. Published 2018 Feb 7. doi:10.3389/fnins.2018.00049

34. Shi N, Li N, Duan X, Niu H. Interaction between the gut microbiome and mucosal immune system. Mil Med Res. 2017;4:14. Published 2017 Apr 27. doi:10.1186/s40779-017-0122-9

35. Fung TC, Olson CA, Hsiao EY. Interactions between the microbiota, immune and nervous systems in health and disease. Nat Neurosci. 2017;20(2):145-155. doi:10.1038/nn.4476

36. Rea K, Dinan TG, Cryan JF. The microbiome: A key regulator of stress and neuroinflammation. Neurobiol Stress. 2016;4:23-33. Published 2016 Mar 4. doi:10.1016/j.ynstr.2016.03.001

37. Sherwin E, Sandhu KV, Dinan TG, Cryan JF. May the Force Be With You: The Light and Dark Sides of the Microbiota-Gut-Brain Axis in Neuropsychiatry. CNS Drugs. 2016;30(11):10191041. doi:10.1007/s40263-016-0370-3

38. Sun M, Wu W, Liu Z, Cong Y. Microbiota metabolite short chain fatty acids, GPCR, and inflammatory bowel diseases. J Gastroenterol. 2017;52(1):1-8. doi:10.1007/s00535-016-1242-9

39. Lin MY, de Zoete MR, van Putten JP, Strijbis K. Redirection of Epithelial Immune Responses by Short-Chain Fatty Acids through Inhibition of Histone Deacetylases. Front Immunol. 2015;6:554. Published 2015 Nov 3. doi:10.3389/fimmu.2015.00554

40. Morrison DJ, Preston T. Formation of short chain fatty acids by the gut microbiota and their impact on human metabolism. Gut Microbes. 2016;7(3):189-200. doi:10.1080/19490976.2015.1134082

41. Ohira H, Tsutsui W, Fujioka Y. Are Short Chain Fatty Acids in Gut Microbiota Defensive Players for Inflammation and Atherosclerosis?. J Atheroscler Thromb. 2017;24(7):660-672. doi:10.5551/jat.RV17006

42. Lach G, Schellekens H, Dinan TG, Cryan JF. Anxiety, Depression, and the Microbiome: A Role for Gut Peptides. Neurotherapeutics. 2018;15(1):36-59. doi:10.1007/s13311-017-0585-0

43. Petra AI, Panagiotidou S, Hatziagelaki E, Stewart JM, Conti P, Theoharides TC. GutMicrobiota-Brain Axis and Its Effect on Neuropsychiatric Disorders With Suspected Immune Dysregulation. Clin Ther. 2015;37(5):984-995. doi:10.1016/j.clinthera.2015.04.002

44. Wong ML, Inserra A, Lewis MD, Mastronardi CA, Leong L et al. Inflammasome signaling affects anxiety- and depressive-like behavior and gut microbiome composition. Mol Psychiatry. 2016;21(6):797-805. doi:10.1038/mp.2016.46

45. Diaz Heijtz R, Wang S, Anuar F, Qian Y, Björkholm B et al. Normal gut microbiota modulates brain development and behavior. Proc Natl Acad Sci U S A. 2011;108(7):3047-3052. doi:10.1073/pnas.1010529108 
46. Bokulich NA, Chung J, Battaglia T, Henderson N, Jay M et al. Antibiotics, birth mode, and diet shape microbiome maturation during early life. Sci Transl Med. 2016;8(343):343ra82. doi:10.1126/scitranslmed.aad7121

47. Kim YK, Shin C. The Microbiota-Gut-Brain Axis in Neuropsychiatric Disorders: Pathophysiological Mechanisms and Novel Treatments. Curr Neuropharmacol. 2018;16(5):559-573. doi:10.2174/1570159X15666170915141036

48. Manderino L, Carroll I, Azcarate-Peril MA, Rochette A, Heinberg L et al. Preliminary Evidence for an Association Between the Composition of the Gut Microbiome and Cognitive Function in Neurologically Healthy Older Adults. J Int Neuropsychol Soc. 2017;23(8):700-705. doi:10.1017/S1355617717000492

49. Kiecolt-Glaser JK, Derry HM, Fagundes CP. Inflammation: depression fans the flames and feasts on the heat. Am J Psychiatry. 2015;172(11):1075-1091. doi:10.1176/appi.ajp.2015.15020152

50. Galley JD, Bailey MT. Impact of stressor exposure on the interplay between commensal microbiota and host inflammation. Gut Microbes. 2014;5(3):390-396. doi:10.4161/gmic.28683

51. Huo R, Zeng B, Zeng L, Cheng K, Li B et al. Microbiota Modulate Anxiety-Like Behavior and Endocrine Abnormalities in Hypothalamic-Pituitary-Adrenal Axis. Front Cell Infect Microbiol. 2017;7:489. Published 2017 Nov 30. doi:10.3389/fcimb.2017.00489

52. Borrelli L, Aceto S, Agnisola C, De Paolo S, Dipineto L et al. Probiotic modulation of the microbiota-gut-brain axis and behaviour in zebrafish. Sci Rep. 2016;6:30046. Published 2016 Jul 15. doi:10.1038/srep30046

53. Sharon G, Cruz NJ, Kang DW, Gandal MJ, Wang B et al. Human Gut Microbiota from Autism Spectrum Disorder Promote Behavioral Symptoms in Mice. Cell. 2019;177(6):1600-1618.e17. doi:10.1016/j.cell.2019.05.004

54. den Besten G, van Eunen K, Groen AK, Venema K, Reijngoud DJ, Bakker BM. The role of short-chain fatty acids in the interplay between diet, gut microbiota, and host energy metabolism. $J$ Lipid Res. 2013;54(9):2325-2340. doi:10.1194/jlr.R036012

55. Lam YY, Maguire S, Palacios T, Caterson ID. Are the Gut Bacteria Telling Us to Eat or Not to Eat? Reviewing the Role of Gut Microbiota in the Etiology, Disease Progression and Treatment of Eating Disorders. Nutrients. 2017;9(6):602. Published 2017 Jun 14. doi:10.3390/nu9060602

56. Glenny EM, Bulik-Sullivan EC, Tang Q, Bulik CM, Carroll IM. Eating Disorders and the Intestinal Microbiota: Mechanisms of Energy Homeostasis and Behavioral Influence. Curr Psychiatry Rep. 2017;19(8):51. doi:10.1007/s11920-017-0797-3

57. Smyth AM, Lawrie SM. The neuroimmunology of schizophrenia. Clin Psychopharmacol Neurosci. 2013;11(3):107-117. doi:10.9758/cpn.2013.11.3.107

58. Fatemi SH, Folsom TD. The neurodevelopmental hypothesis of schizophrenia, revisited. Schizophr Bull. 2009;35(3):528-548. doi:10.1093/schbul/sbn187

59. Jaaro-Peled H, Hayashi-Takagi A, Seshadri S, Kamiya A, Brandon NJ, Sawa A. Neurodevelopmental mechanisms of schizophrenia: understanding disturbed postnatal brain maturation through neuregulin-1-ErbB4 and DISC1. Trends Neurosci. 2009;32(9):485-495. doi:10.1016/j.tins.2009.05.007

60. Bonnín CDM, Reinares M, Martínez-Arán A, Jiménez E, Sánchez-Moreno J et al. Improving Functioning, Quality of Life, and Well-being in Patients With Bipolar Disorder. Int $J$ Neuropsychopharmacol. 2019;22(8):467-477. doi:10.1093/ijnp/pyz018

61. Harrison PJ, Geddes JR, Tunbridge EM. The Emerging Neurobiology of Bipolar Disorder. Trends Neurosci. 2018;41(1):18-30. doi:10.1016/j.tins.2017.10.006

62. Dickerson F, Severance E, Yolken R. The microbiome, immunity, and schizophrenia and bipolar disorder. Brain Behav Immun. 2017;62:46-52. doi:10.1016/j.bbi.2016.12.010 
63. Lichtenstein P, Yip BH, Björk C, Pawitan Y, Cannon TD et al. Common genetic determinants of schizophrenia and bipolar disorder in Swedish families: a population-based study. Lancet. 2009;373(9659):234-239. doi:10.1016/S0140-6736(09)60072-6

64. Nguyen TT, Kosciolek T, Eyler LT, Knight R, Jeste DV. Overview and systematic review of studies of microbiome in schizophrenia and bipolar disorder. J Psychiatr Res. 2018;99:50-61. doi:10.1016/j.jpsychires.2018.01.013

65. Zheng P, Zeng B, Liu M, Chen J, Pan Jet al. The gut microbiome from patients with schizophrenia modulates the glutamate-glutamine-GABA cycle and schizophrenia-relevant behaviors in mice [published correction appears in Sci Adv. 2019 Jun 21;5(6):eaay2759]. Sci Adv. 2019;5(2):eaau8317. Published 2019 Feb 6. doi:10.1126/sciadv.aau8317

66. Liang W, Huang Y, Tan X, Wu J, Duan J et al. Alterations Of Glycerophospholipid And Fatty Acyl Metabolism In Multiple Brain Regions Of Schizophrenia Microbiota Recipient Mice. Neuropsychiatr Dis Treat. 2019;15:3219-3229. Published 2019 Nov 19. doi:10.2147/NDT.S225982

67. Hu S, Li A, Huang T, Lai J, Li J et al. Gut Microbiota Changes in Patients with Bipolar Depression. Adv Sci (Weinh). 2019;6(14):1900752. Published 2019 May 15. doi:10.1002/advs.201900752

68. Aizawa E, Tsuji H, Asahara T, Takahashi $\mathrm{T}$, Teraishi $\mathrm{T}$ et al. Bifidobacterium and Lactobacillus Counts in the Gut Microbiota of Patients With Bipolar Disorder and Healthy Controls. Front Psychiatry. 2019;9:730. Published 2019 Jan 18. doi:10.3389/fpsyt.2018.00730 